\title{
General Theory of Optimal Trajectory for Rocket Flight in a Resisting Medium ${ }^{1}$
}

\author{
N. X. $\mathrm{VINH}^{2}$ \\ Communicated by A. Miele
}

\begin{abstract}
This paper considers the problem of optimizing the flight trajectory of a rocket vehicle moving in a resisting medium and in a general gravitational force field. General control laws for the lift, the bank angle, and the thrusting program are obtained in terms of the primer vector, the adjoint vector associated to the velocity vector. Additional relations for the case of variable thrusting and integrals of motion for flight at maximum lift-to-drag ratio and flight in a constant gravitational field are obtained.
\end{abstract}

\section{Introduction}

The problem of determining optimal trajectories for a rocketpowered vehicle flying inside the atmosphere of a planet has received considerable attention in recent years. In general, it was assumed that the gravitational field is uniform. With the advent of the maneuverable hypervelocity vehicle and long-range lifting rocket, it is necessary to remove that restriction. With the classical results of Lawden (Ref. 1) and Breakwell (Ref. 2) for flight in a vacuum; Leitmann. (Ref. 3), Miele (Ref. 4), and Bryson and Lele (Ref. 5) for flight inside an atmosphere; and the recent contributions by Busemann, Vinh, and Kelley (Ref. 6), and Vinh (Ref. 7) it is now possible to derive the most general results concerning the optimal trajectories of a lifting vehicle powered by a rocket engine and subject to aerodynamic forces in a general graviational force field.

Consider the motion of a vehicle under such conditions. At the time $t$, the state of the vehicle is defined by the position vector $\mathbf{r}(t)$,

\footnotetext{
1 This work was supported by Air Force Grant No. AFOSR-71 -2129.

${ }^{2}$ Professor, Department of Aerospace Engineering, The University of Michigan, Ann Arbor, Michigan.
} 


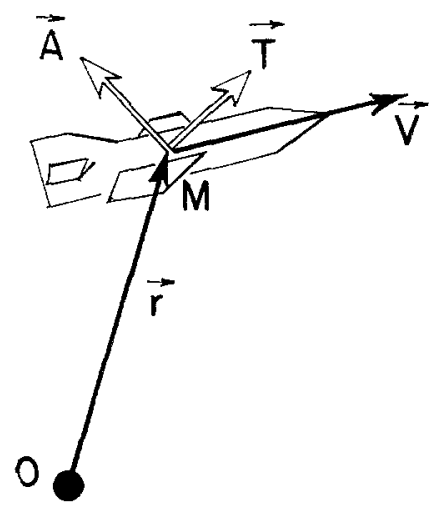

Fig. 1. State and control variables.

the velocity vector $\mathbf{V}(t)$, and the instantaneous mass $m(t)$. The flight path is controlled by a thrusting force $\mathbf{T}$ and the aerodynamic force $\mathbf{A}$ (Fig. 1). It is assumed that the direction of the thrust can be orientated arbitrarily but, at any instant $t$, the aerodynamic force is constrained in such a way that the terminus of the vector $\mathbf{A}$ is located on a certain surface that varies as function of the time.

More specifically, we consider a coordinate system centered at the center of mass $M$ of the vehicle with an axis constantly aligned with the velocity $\mathbf{V}$ (Fig. 2). Then, the control vector $\mathbf{T}$ can be taken such that its terminus is inside the solid sphere centered at $M$ and with radius

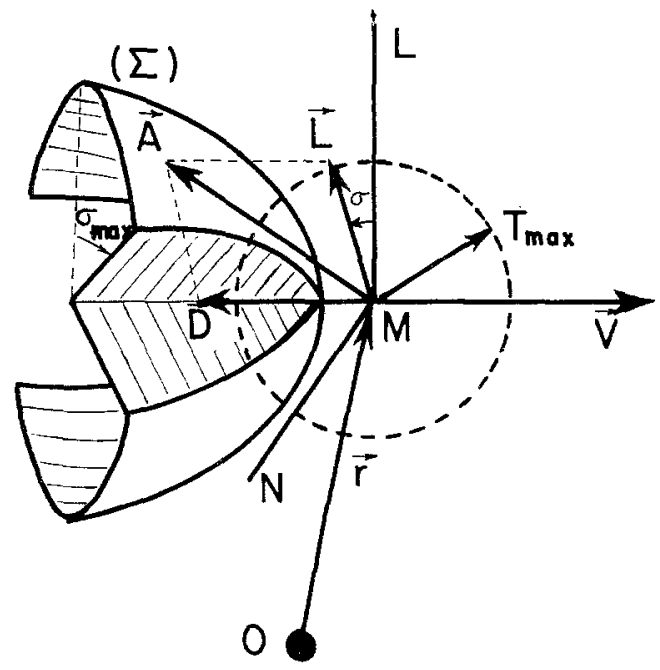

Fig. 2. Control space. 
$T_{\max }$. Hence, while the direction of $\mathbf{T}$ can be taken arbitrarily, its magnitude is bounded by

$$
0 \leqslant T \leqslant T_{\max } .
$$

The aerodynamic force $\mathbf{A}$ is decomposed into a drag force $\mathbf{D}$, always in the opposite direction to $\mathbf{V}$, and a lift force $\mathbf{L}$ orthogonal to it. The constraint on $\mathbf{A}$ is expressed in the form of a lift-drag relationship

$$
D=D(L, V, \mathbf{r}) \text {. }
$$

We shall use the usual assumption

$$
L=\frac{1}{2} \rho S C_{L} V^{2}, \quad D=\frac{1}{2} \rho S C_{D} V^{2},
$$

where $S$ is a reference area, and $\rho$ is the mass density of the atmosphere (a function of the position vector $\mathbf{r}$ ). $C_{L}$ and $C_{D}$ are the lift and the drag coefficients, which for hypervelocity flight are independent of the Mach number and the Reynolds number. If the vehicle has a plane of symmetry, in coordinated flight, $\mathbf{A}$ and $\mathbf{V}$ are in that plane. We define the bank angle $\sigma$ as the angle between the osculating plane $(r, V)$ and the plane of symmetry $(\mathbf{A}, \mathbf{V})$ of the vehicle. We take the $L$-axis in the osculating plane and the $\mathrm{N}$-axis perpendicular to it in such a way that the axes $V L N$ form a right-handed orthogonal axis system. In this system, the drag polar as defined by (2) is a surface of revolution about the axis V. By the assumption (3), the lift-drag relation can be replaced by the relation between the aerodynamic coefficients

$$
C_{D}=C_{D}\left(C_{L}\right)
$$

Because of this constraint, the aerodynamic force $\mathbf{A}$ is defined by its magnitude and the bank angle $\sigma$.

In practice, the lift coefficient is bounded, and so is the bank angle. Hence, for the aerodynamic force control we shall use $C_{L}$ and $\sigma$ or, equivalently, the angle of attack and the bank angle. They are subject to the constraints

$$
0 \leqslant C_{L} \leqslant C_{L \max }
$$

and

$$
0 \leqslant|\sigma| \leqslant \sigma_{\max }
$$

The surface of revolution $\Sigma$ which defines the control space for $\mathbf{A}$ is restricted to two symmetric portions of surface. By using a homothety with center $M$ and ratio $\left(\frac{1}{2} \rho S V^{2}\right)^{-1}$, we can replace the varying space $\Sigma$ by the fixed space $\Sigma^{\prime}$ representing the relation (4), subject to the constraints $(5)-(6)$. 


\section{Equations of Motion}

The motion of the vehicle, considered as a mass point, flying in a general gravitational field and subject to aerodynamic and thrusting forces, is governed by the equation

$$
d \mathbf{r} / d t=\mathbf{V}, \quad d \mathbf{V} / d t=(1 / m)(\mathbf{T}+\mathbf{A})+\mathbf{g}(\mathbf{r}, t), \quad d m / d t=-T / c,
$$

where $c$ is the constant exhaust velocity of the gas ejected from the engine, and $g$ is the acceleration of the gravitational field. The optimal transfer problem is defined as follows.

At the initial time $t=0, \mathbf{r}=\mathbf{r}_{0}, \mathbf{V}=\mathbf{V}_{0}, m=m_{0}$. The vectors $\mathbf{r}_{0}, \mathbf{V}_{0}$ and the scalar $m_{0}$ are prescribed. At the final time $t=t_{f}, \mathbf{r}=\mathbf{r}_{f}$, $\mathbf{V}=\mathbf{V}_{f}$ and $m=m_{f}$. The problem is to find the time histories $\mathbf{T}(t)$ and $\mathbf{A}(t)$ such that some scalar function of the final state is a minimum.

Using the maximum principle, we introduce the adjoint elements $\mathbf{p}_{r}, \mathbf{p}_{V}$, and $p_{m}$ to form the Hamiltonian

$$
H=\mathbf{p}_{r} \cdot \mathbf{V}+(1 / m) \mathbf{p}_{V} \cdot(\mathbf{T}+\mathbf{A})+\mathbf{p}_{V} \cdot \mathbf{g}(\mathbf{r}, t)-\left(p_{m} T / c\right),
$$

where $\mathbf{p}_{r}, \mathbf{p}_{V}$, and $p_{m}$ are defined by the adjoint equations

$$
d \mathbf{p}_{r} / d t=-\partial H / \partial \mathbf{r}, \quad d \mathbf{p}_{V} / d t=-\partial H / \partial \mathbf{V}, \quad d p_{m} / d t=-\partial H / \partial m .
$$

The solution is obtained by integrating the systems of Eqs. (7) and (9), subject to specified end-conditions, and selecting the control $\mathbf{T}$ and $\mathbf{A}$ in their bounded spaces such that at each instant the Hamiltonian defined by ( 8$)$ is an absolute maximum.

\section{Optimal Controls}

The Hamiltonian must be maximized with respect to $\mathbf{T}$ and $\mathbf{A}$. At each instant $t$, the terminus of the vector $\mathbf{T}$ is inside the solid sphere with center $M$ and radius $T_{\max }$, while the terminus of the vector $\mathbf{A}$ is on the portion of the surface of revolution $(\Sigma)$ (Fig. 3).

Consider the vector $\mathbf{p}_{v}$ in the expression (8) for the Hamiltonian. Since $\mathbf{T}$ and $\mathbf{A}$ are independent controls, we first maximize the dot product $\mathbf{p}_{V} \cdot \mathbf{T}$. It is clear that the two vectors $\mathbf{p}_{V}$ and $\mathbf{T}$ must be collinear. Hence, if the engine is operating, it must orientate the thrust in the direction of $\mathbf{p}_{V}$, and we have

$$
\max \left(\mathbf{p}_{V} \cdot \mathbf{T}\right)=p_{V} T,
$$

where $p_{V}$ is the magnitude of the vector $\mathbf{p}_{V}$, called the primer vector. 


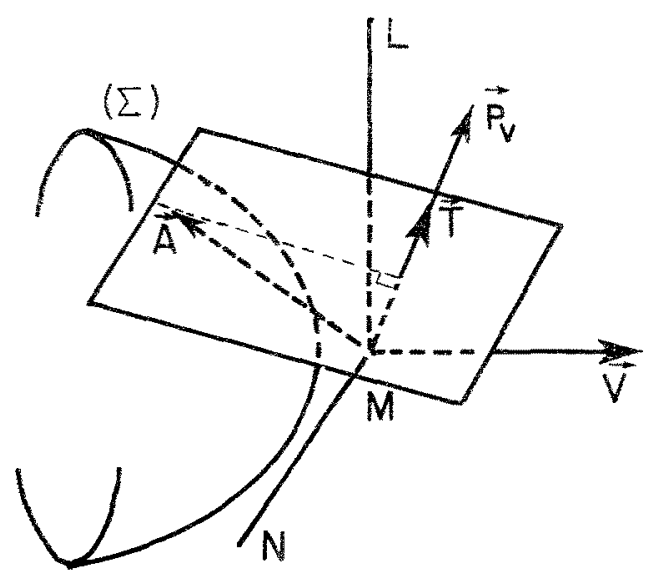

Fig. 3. Optimal controls.

Next, it is necessary to select the aerodynamic force $\mathbf{A}$ such that the dot product $\mathbf{p}_{V} \cdot \mathbf{A}$ is maximized. If the surface $\Sigma$ is not constrained, this is obtained by taking the terminus of the vector $\mathbf{A}$ at the contact point of the plane tangent to $\Sigma$, while orthogonal to $\mathbf{p}_{V}$. To construct this point, we consider the plane defined by $\mathbf{V}$ and $\mathrm{p}_{V}$. This plane intersects $\Sigma$ along a meridian (Fig. 4), which is precisely the drag polar defined by the relation (2). Let $\epsilon$ be the angle between $\mathbf{V}$ and $\mathbf{p}_{V}$. We note that this angle is also the angle between $V$ and the optimal thrust direction. Since the rotated $L$-axis is perpendicular to $V$, the tangent

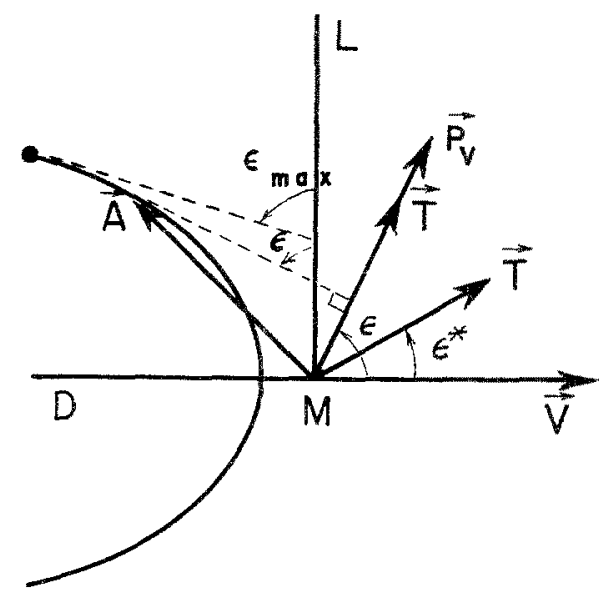

Fig. 4. Optimal lift control. 
to the drag polar and orthogonal to $\mathbf{p}_{V}$ makes the same angle $\epsilon$ with the $L$-axis. Hence from the geometry of the figure

$$
\tan \epsilon=\partial D / \partial L
$$

Since $\Sigma$ is a surface of revolution, a plane perpendicular to the plane $\left(\mathbf{V}, \mathbf{p}_{V}\right)$ along the tangent described is also tangent to $\Sigma$. Hence, the optimal control law for the lift is given by (11). Miele first obtained this relation (Ref. 8) for two-dimensional flight in a constant gravitational field. If we assume that the lift and drag forces are proportional to $C_{L}$ and $C_{D}$ by the same factor, relation (11) can be replaced by

$$
\tan \epsilon=\partial C_{D} / \partial C_{L} .
$$

From the construction of the optimal aerodynamic force, it has been shown that the three vectors $\mathbf{p}_{p}, \mathbf{V}$, and $\mathbf{A}$ are in the same plane. Mathematically, this is expressed by the relation

$$
\left(\mathbf{V} \times \mathbf{p}_{V}\right) \cdot \mathbf{A}=\mathbf{0} .
$$

Hence, (11) and (13) together define the optimal lift and bank programs when the aerodynamic force control is not constrained.

From Fig. 4, it is seen that, if Ineqs. (5) are enforced, then when

$$
\epsilon \geqslant \epsilon_{\max },
$$

where $\epsilon_{\max }$ is the angle between the tangent at the point of maximum. lift [or, if (12) is used, the point of maximum lift coefficient] and the lift axis, in this case the optimal lift program as defined by (12) must be replaced by

$$
\text { optimal } C_{L}=C_{L \max } \text {. }
$$

The optimal relation (13) is valid when the bank angle is not constrained. Let $\sigma$ be the angle between the plane $\left(\mathbf{V}, \mathbf{p}_{V}\right)$ and the osculating plane $(\mathbf{r}, \mathbf{V})$. If the inequalities (6) are enforced, then when

$$
\sigma \geqslant \sigma_{\max }
$$

the terminus of the vector $\mathbf{A}$ must be selected along the drag polar, intersection of the plane $\sigma_{\max }$ and the surface $\Sigma$. In this case, relation (13) for the optimal bank angle must be replaced by (Fig. 5)

$$
\text { optimal } \sigma=\sigma_{\max } \text {. }
$$

Let $\mathbf{p}_{V}{ }^{\prime}$ be the projection of the vector $\mathbf{p}_{V}$ into the plane $\sigma_{\max }$. We have

$$
\mathbf{p}_{V}=\mathbf{p}_{V}^{\prime}+\mathbf{p}_{V}^{\prime \prime}
$$




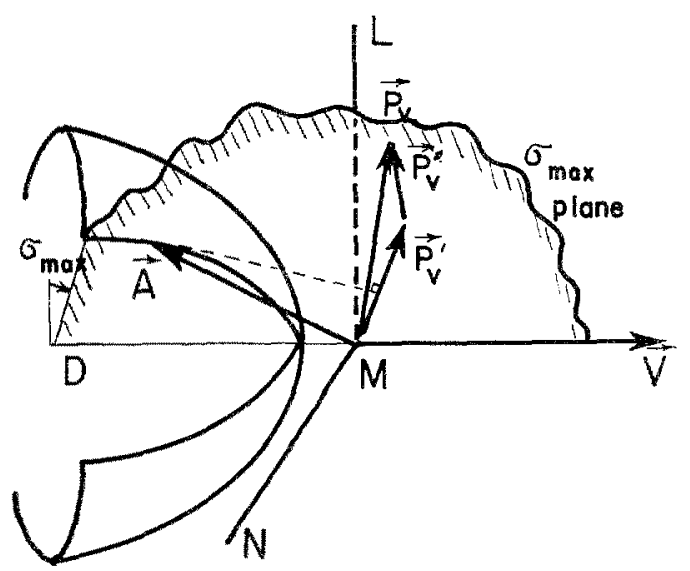

Fig. 5. Optimal hift for maximum bank angle.

where $\mathbf{p}_{V}^{\prime \prime}$ is the component of $\mathbf{p}_{V}$ orthogonal to the plane containing the optimal aerodynamic force $\mathbf{A}$. Then, $\mathbf{p}_{V} \cdot \mathbf{A}=\mathbf{p}_{V}{ }^{\prime} \cdot \mathbf{A}$. Hence, the relation giving the optimal lift control (11) must be replaced by

$$
\tan \epsilon^{\prime}=\partial D / \partial L
$$

where $\epsilon^{\prime}$ is the angle between $\mathbf{V}$ and the vector $\mathbf{p}_{V}{ }^{\prime}$, projection of $\mathbf{p}_{V}$ on the plane $\sigma_{\max }$.

It remains to consider the optimal thrust magnitude. Using (10), we rewrite the Hamiltonian as

$$
H=\mathbf{p}_{r} \cdot \mathbf{V}+\mathbf{p}_{V} \cdot[\mathbf{g}+(1 / m) \mathbf{A}]+(T / m)\left[p_{V}-\left(m p_{m} / c\right)\right] .
$$

Define the switching function

$$
K=p_{V}-\left(m p_{m} / c\right) .
$$

Then, to maximize $H$, the following rules apply:

$$
\begin{aligned}
& \text { if } K>0, \text { we select } T=T_{\max } \text { (boosting phase); } \\
& \begin{array}{ll}
\text { if } K<0, & \text { we select } T=0 \text { (coasting phase); } \\
\text { if } K=0 \quad \text { for a finite time interval, } \\
\text { we select } T=\text { variable (sustaining phase). }
\end{array}
\end{aligned}
$$

\section{Remarks}

(i) We notice that if the gravitational field is time independent, the Hamiltonian $H$ is a constant of motion. This constant remains the 
same throughout the entire trajectory. Then, if the optimal trajectory contains a coasting or a sustaining arc, the constant can be evaluated at any point along these arcs using the reduced form for the Hamiltonian

$$
H=\mathbf{p}_{r} \cdot \mathbf{V}+\mathbf{p}_{V} \cdot[\mathbf{g}+(1 / m) \mathbf{A}] .
$$

(ii) In the case where the direction of the thrust is constrained, then the optimal thrust may not be directed along the primer vector. More specifically, let $\epsilon^{*}$ be the maximum angle between the velocity and the thrust (Fig. 4). If

$$
\epsilon \geqslant \epsilon^{*}
$$

the optimal thrust direction is given by $\epsilon^{*}$, and relation $(10)$ must be replaced by

$$
\mathbf{p}_{V} \cdot \mathbf{T}=p_{V} T \cos \left(\epsilon-\epsilon^{*}\right) .
$$

In particular, when the thrust is always in the direction of the velocity, $\epsilon^{*}=0$ and

$$
\mathbf{p}_{V} \cdot \mathbf{T}=p_{V} T \cos \epsilon .
$$

(iii) The adjoint components being continuous, by the first integral (20) for the case of time-invariant gravitational field, a discontinuity in the mass flow (that is, a jump in the thrust control) is possible only when

$$
K=0 \text {. }
$$

Hence, this relation defines a junction point between different types of optimal arcs. On the other hand, a discontinuity in the aerodynamic force control is possible only when

$$
\mathbf{p}_{V} \cdot \mathbf{A}_{-}=\mathbf{p}_{V} \cdot \mathbf{A}_{+} \cdot
$$

Because of the symmetry of the polar surface $\Sigma$, this requires that, at the instant of switching, either $\mathbf{p}_{V} \cdot \mathbf{A}_{-}=\mathbf{p}_{V} \cdot \mathbf{A}_{+}=0$ or, in the case of flying at maximum bank angle, $\mathbf{p}_{V}{ }^{\prime} \cdot \mathbf{A}_{-}=\mathbf{p}_{V}{ }^{\prime} \cdot \mathbf{A}_{+}=0$. Since $\mathbf{p}_{Y}$ and $\mathbf{p}_{V}^{\prime}$ are continuous, any discontinuity in $\mathbf{A}$ requires that $\mathbf{p}_{V}=0$.

(iv) The relation

$$
\mathrm{p}_{V} \cdot \mathbf{A}=0
$$

for $\mathbf{p}_{V} \neq 0$ shows that the tangent to the drag polar at the optimal operating point passes through the origin $M$ of the coordinate system. The vehicle is flying at maximum lift-to-drag ratio (Fig. 4).

The results of this section are collected in Table 1 . 
Table 1. Optimal aerodynamic and thrusting controls.

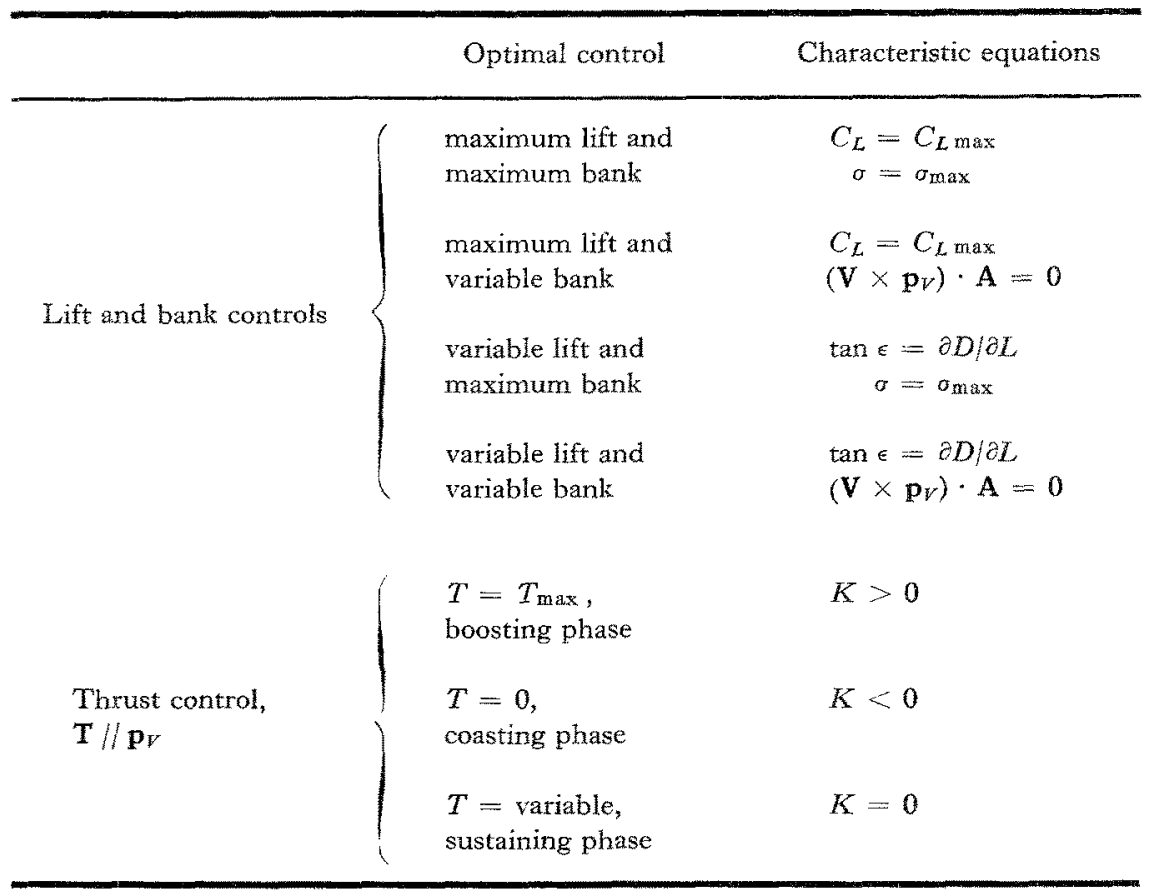

\section{Integrals of Motion}

If the lift and drag forces are of the form given by (3), we can write the adjoint equations (9) explicitly as

$$
\begin{aligned}
& d \mathbf{p}_{r} / d t=-\nabla\left(\mathbf{p}_{V} \cdot \mathbf{g}\right)-(1 / m) \nabla\left(\mathbf{p}_{V} \cdot \mathbf{A}\right), \\
& d \mathbf{p}_{V} / d t=-\mathbf{p}_{r}-\left[2\left(\mathbf{p}_{V} \cdot \mathbf{A}\right) / m V^{2}\right] \mathbf{V}, \\
& d p_{m} / d t=\left(1 / m^{2}\right)\left(\mathbf{p}_{V} \cdot \mathbf{A}\right)+\left(1 / m^{2}\right) p_{V} T,
\end{aligned}
$$

where $\nabla$ is the gradient operator in the position-coordinate directions. When the aerodynamic forces are not present, there exists a number of integrals of motion (Ref. 9). It has been shown that, in this case, the primer vector can be obtained for a general, time-invariant, central gravitational field (Ref. 7) when the vehicle is along a coasting arc. Also, when the vehicle is along a variable-thrust arc, the planar solution can be obtained (Ref. 1). 
For flight in a resisting medium, because of the nonconservative nature of the aerodynamic forces and the nonlinearity of the full equations considered in this paper, integrals of motion can only be formulated under some restrictive assumptions.

First, for a time-invariant gravitational field, we always have the Hamiltonian constant (20). When the vehicle is along a sustaining arc, we have the additional relation

$$
p_{V}-m p_{m} / c=0 .
$$

This relation being satisfied for a finite time interval, we can take its derivative to have

$$
\begin{aligned}
d p_{V} / d t & =(m / c)\left(d p_{m} / d t\right)+\left(p_{m} / c\right)(d m / d t) \\
& =(1 / m c)\left(\mathbf{p}_{V} \cdot \mathbf{A}\right)+(1 / m c) p_{V} T-\left(p_{m} T / c^{2}\right) .
\end{aligned}
$$

Using (31), we have

$$
d p_{V} / d t=(1 / m c)\left(\mathbf{p}_{V} \cdot \mathbf{A}\right) .
$$

Now, we consider

$$
(d / d t)\left(\mathbf{p}_{V} \cdot \mathbf{p}_{V}\right)=2 p_{V}\left(d p_{V} / d t\right)=2 \mathbf{p}_{V} \cdot\left(d \mathbf{p}_{V} / d t\right),
$$

or

$$
\left(m p_{m} / c\right)\left(d p_{V} / d t\right)=-\mathbf{p}_{V} \cdot \mathbf{p}_{r}-\left[2\left(\mathbf{p}_{V} \cdot \mathbf{A}\right) / m V^{2}\right]\left(\mathbf{p}_{V} \cdot \mathbf{V}\right) .
$$

By eliminating $d p_{v} / d t$ between Eqs. (32)-(33), we have another relation for sustaining arc, namely,

$$
-\mathbf{p}_{V} \cdot \mathbf{p}_{r}=\left(\mathbf{p}_{V} \cdot \mathbf{A}\right)\left\{\left[2\left(\mathbf{p}_{V} \cdot \mathbf{V}\right) / m V^{2}\right]+\left(p_{m} / c^{2}\right)\right\} .
$$

The thrust magnitude control would appear upon taking the derivative of this last equation. This step would remove the indetermination for the thrust magnitude control due to the vanishing of the switching function $K$. The two relations (31) and (34) allow the elimination of two differential equations for the adjoint components and make possible a complete solution for sustaining flight when other integrals are available or when the number of state variables is reduced.

We next consider the differential

$$
(d / d t)\left(m p_{m}\right)=m\left(d p_{m} / d t\right)+p_{m}(d m / d t) .
$$

Replacing the derivatives on the right-hand side by their expressions from the state and adjoint equations, we have

$$
(d / d t)\left(m p_{m}\right)=(1 / m)\left(\mathbf{p}_{V} \cdot \mathbf{A}\right)+(T / m)\left[p_{V}-\left(m p_{m} / c\right)\right] .
$$


There is an important class of optimal trajectory, namely the equilibrium glide trajectory where the vehicle is flown at maximum lift-to-drag ratio. The condition for this particular trajectory is expressed by

$$
\mathbf{p}_{V} \cdot \mathbf{A}=0 .
$$

Then, along this trajectory, the adjoint system (30) becomes

$$
d \mathbf{p}_{r} / d t=-\nabla\left(\mathbf{p}_{V} \cdot \mathbf{g}\right), \quad d \mathbf{p}_{V} / d t=-\mathbf{p}_{r}, \quad d p_{m} / d t=\left(1 / m^{2}\right) p_{V} T,
$$

with the Hamiltonian having the reduced form

$$
H=\mathbf{p}_{r} \cdot \mathbf{V}+\mathbf{p}_{V} \cdot \mathbf{g}+(T / m)\left[p_{r}-\left(m p_{m} / c\right)\right] .
$$

By eliminating $\mathbf{p}_{r}$ between the first two equations (37), we have the equation for the primer vector

$$
d^{2} \mathbf{p}_{V} / d t^{2}=\nabla\left(\mathbf{g} \cdot \mathbf{p}_{V}\right)
$$

Equations (37)-(39) are identical to the variational equations for rocket flight when there is no aerodynamic force involved. However, a complete similarity does not prevail, since the aerodynamic force $\mathbf{A}$ is still present in the state equations (7). Nevertheless, the assumption of maximum lift-to-drag ratio allows the formulation of some additional first integrals. The differential expression (35) now becomes

$$
(d / d t)\left(m p_{m}\right)=(T / m)\left[p_{V}-\left(m p_{m} / c\right)\right] .
$$

Along a coasting arc, $T=0$, and we have $m p_{m}=$ const. Or, since the mass does not vary along a coasting arc,

$$
p_{m}=\text { const. }
$$

This integral can also be obtained by integrating directly the last equation (37).

Along a sustaining arc, we also have

$$
m p_{m}=\text { const. }
$$

Since $K=0$ along this arc, by (21) we deduce

$$
p_{V}=\text { const. }
$$

If we write this equation as $\mathrm{p}_{V} \cdot \mathbf{p}_{V}=$ const and take its derivative, then, with the aid of the second equation (37), we have

$$
\mathbf{p}_{V} \cdot \mathbf{p}_{r}=0 \text {. }
$$


This relation can also be obtained immediately from (34). Hence, for optimal maximum lift-to-drag ratio flight along a sustaining arc, the thrust is in the direction of the primer vector. This vector has constant magnitude and is orthogonal to $\mathbf{p}_{r}$.

Furthermore, from Fig. 4, the angle $\epsilon$ between $\mathbf{V}$ and $\mathbf{p}_{v}$ is constant. This result is valid for both the boosting arc and the sustaining arc. Also from Fig. 4, it is seen that, whenever the engine is operating, the thrust is in the plane of $\mathbf{V}$ and $\mathbf{A}$ and is directed orthogonally to the aerodynamic force.

Finally, still under the assumption of maximum lift-to-drag ratio, we consider a central force field of the form

$$
\mathbf{g}=-\left(\mu / r^{n}\right)(\mathbf{r} / r)
$$

a particular case of which is the familiar Newtonian, inverse-square force field. We rewrite the first two equations (30) as

$$
d \mathbf{p}_{r} / d t=\left(\mu / r^{n+1}\right)\left\{\mathbf{p}_{V}-\left[(n+1)\left(\mathbf{p}_{V} \cdot \mathbf{r}\right) \mathbf{r} / r^{2}\right]\right\}, \quad d \mathbf{p}_{V} / d t=-\mathbf{p}_{r} .
$$

We now consider the differential

$$
\begin{aligned}
& (d / d t)\left(\mathbf{p}_{V} \cdot \mathbf{V}-a \mathbf{p}_{r} \cdot \mathbf{r}\right) \\
& \quad=\left(d \mathbf{p}_{V} / d t\right) \cdot \mathbf{V}+\mathbf{p}_{V} \cdot(d \mathbf{V} / d t)-a\left(d \mathbf{p}_{r} / d t\right) \cdot \mathbf{r}-a \mathbf{p}_{r} \cdot(d \mathbf{r} / d t),
\end{aligned}
$$

where $a=2 /(n-1)$. Using the state equations (7) and the reduced adjoint equations (46) together with the relations $(10),(36),(38)$, and (45), we have

$(d / d t)\left(\mathbf{p}_{V} \cdot \mathbf{V}-a \mathbf{p}_{r} \cdot \mathbf{r}\right)=-b H+b(T / m)\left[p_{V}-\left(m p_{m} / c\right)\right]+\left(p_{V} T / m\right)$,

where $b=(n+1) /(n-1)$.

Along a coasting arc, $T=0$ and we have the integral

$$
\mathbf{p}_{V} \cdot \mathbf{V}-a \mathbf{p}_{r} \cdot \mathbf{r}=-b H t+k,
$$

where $k$ is a constant of integration.

Along a sustaining arc, where $p_{V}$ is constant and $K=0$, we have the integral

$$
\mathbf{p}_{V} \cdot \mathbf{V}-a \mathbf{p}_{r} \cdot \mathbf{r}=-b H t+p_{V} \int_{0}^{t}(T / m) d t+k
$$

We notice that the integral in the last equation represents the characteristic velocity $\Delta V$ spent since the initial time of thrusting. Hence, if impulsive thrust is allowed, since during the infinitesimal time for 
thrusting the switching function $K$ is zero, the first integral (49) is also valid for impulsive thrust. We conclude that, for flight at maximum lift-to-drag ratio, with an engine capable of delivering infinite thrust magnitude, we have, over the entire optimal trajectory, the integral

$$
\mathbf{p}_{V} \cdot \mathbf{V}-a \mathbf{p}_{r} \cdot \mathbf{r}=-b H t+p_{V} \Delta V+k
$$

where $\Delta V$ is the total characteristic velocity. The gravitational force field considered is the one defined by (45).

\section{Optimal Trajectories in a Uniform Field}

As in the case of rocket flight in a vacuum, the general equations are greatly simplified when the gravitational field is uniform. In these circumstances, $\mathrm{g}$ is constant and we have the adjoint equations

$$
\begin{aligned}
& d \mathbf{p}_{V} / d t=-(1 / m) \nabla\left(\mathbf{p}_{V} \cdot \mathbf{A}\right), \\
& d \mathbf{p}_{V} / d t=-\mathbf{p}_{r}-\left[2\left(\mathbf{p}_{V} \cdot \mathbf{A}\right) / m V^{2}\right] \mathbf{V}, \\
& d p_{m} / d t=\left(\mathbf{1} / m^{2}\right)\left(\mathbf{p}_{V} \cdot \mathbf{A}\right)+\left(\mathbf{1} / m^{2}\right) p_{V} T .
\end{aligned}
$$

If the flight is effectuated at maximum lift-to-drag ratio, we have

$$
d^{2} \mathbf{p}_{V} / d t^{2}=0
$$

and the general solution for the primer is evidently

$$
\mathrm{p}_{V}=\xi_{t}+\eta
$$

where $\xi$ and $\eta$ are constant vectors. The solution can be obtained in a fairly complete form.

For the case of variable lift program, we take the $z$-axis along the opposite direction to the constant gravity and assume that the atmospheric mass density is solely a function of the altitude. Then, in component form, along the $x$-axis and the $y$-axis, the first equation (51) gives

$$
d p_{r_{x}} / d t=0, \quad d p_{r_{y}} / d t=0 .
$$

These equations give us two integrals

$$
p_{r_{x}}=\text { const, } \quad p_{r_{y}}=\text { const. }
$$

If the longitudinal and the lateral ranges are free, the above constants are zero and subsequently will further simplify the set of adjoint equations (51). 


\section{Conclusions}

This paper considers the problem of optimizing the flight trajectory of a rocket vehicle moving in a resisting medium, in a general gravitational force field. General control laws for the lift, the bank angle, and the thrusting program have been obtained in terms of the primer vector, the adjoint vector $\mathrm{p}_{V}$ associated to the velocity vector. A number of integrals of motion have been formulated for particular cases of special interest.

\section{References}

1. Lawden, D. F., Optimal Trajectories for Space Navigation, Butterworths, London, 1963.

2. Breakwell, J. V., The Optimization of Trajectories, SIAM Journal on Applied Mathematics, Vol. 7, No. 2, 1959.

3. Leitmann, G., A Calculus of Variations Solution of Goddard's Problem, Astronautica Acta, Vol. 2, No. 2, 1956.

4. Miese, A., General Variational Theory of the Flight Paths of Rocket-Powered Aircraft, Missile, and Satellite Carriers, Astronautica Acta, Vol. 4, No.4, 1958.

5. Bryson, A. E., and Lele, M. M., Minimum Fuel Lateral Turns at Constant Altitude, AIAA Journal, Vol. 7, No. 3, 1969.

6. Buseman, A., Vinh, N. X., and Kelley, G. F., Optimum Maneuvers of a Skip Vehicle with Bounded Lift Constraints, Journal of Optimization Theory and Applications, Vol. 3, No. 4, 1969.

7. VInH, N. X., Integration of the Primer Vector in a Central Force Field, Journal of Optimization Theory and Applications, Vol. 9, No. 1, 1972.

8. Mrele, A., The Calculus of Variations in Applied Aerodynamics and Flight Mechanics, Optimization Techniques, Edited by G. Leitmann, Academic Press, New York, 1962.

9. PInEs, S., Constants of the Motion for Optimum Thrust Trajectories in a Central Force Field, AIAA Journal, Vol. 2, No. 11, 1964. 